\title{
Polymerase chain reaction primer sets for the detection of genetically diverse human sapoviruses
}

\author{
Tomoichiro Oka ${ }^{1}$ (Deiji P. Yamamoto ${ }^{2} \cdot$ Nobuhiro Iritani $^{2} \cdot$ Shigenori Sato $^{3} \cdot$ Chika Tatsumi $^{4} \cdot$ Tetsuo Mita $^{4}$. \\ Shunsuke Yahiro ${ }^{5}$. Shinichiro Shibata ${ }^{6} \cdot$ Fang-Tzy Wu $^{7} \cdot$ Hirotaka Takagi $^{8}$
}

Received: 2 May 2020 / Accepted: 22 June 2020 / Published online: 27 July 2020

(c) Springer-Verlag GmbH Austria, part of Springer Nature 2020

\begin{abstract}
Sapoviruses are increasingly being recognized as pathogens associated with gastroenteritis in humans. Human sapoviruses are currently assigned to 18 genotypes (GI.1-7, GII.1-8, GIV.1, and GV.1-2) based on the sequence of the region encoding the major structural protein. In this study, we evaluated 11 polymerase chain reaction (PCR) assays using published and newly designed/modified primers and showed that four PCR assays with different primer combinations amplified all of the tested human sapovirus genotypes using either synthetic DNA or cDNA prepared from human sapovirus-positive fecal specimens. These assays can be used as improved broadly reactive screening tests or as tools for molecular characterization of human sapoviruses.
\end{abstract}

Sapoviruses are among the major pathogens associated with sporadic and outbreaks of acute gastroenteritis in humans of all ages [1]. The level of excretion of the viral genome in feces is usually high $[1,2]$. One of the largest sapovirus outbreaks was likely associated with sapovirus-infected food

Handling Editor: Reimar Johne.

Tomoichiro Oka

oka-t@nih.go.jp

1 Department of Virology II, National Institute of Infectious Diseases, Gakuen 4-7-1, Musashimurayama-shi, Tokyo 208-0011, Japan

2 Division of Microbiology, Osaka Institute of Public Health, Osaka, Japan

3 Division of Virology and Medical Zoology, Chiba Prefectural Institute of Public Health, Chiba, Japan

4 Division of Virology, Shimane Prefectural Institute of Public Health and Environmental Science, Shimane, Japan

5 Department of Microbiology, Kumamoto Prefectural Institute of Public Health and Environmental Science, Kumamoto, Japan

6 Microbiology Department, Nagoya City Public Health Research Institute, Aichi, Japan

7 Center for Research, Diagnostics and Vaccine Development, Taiwan Centers for Disease Control, Taipei, Taiwan

8 Management Department of Biosafety and Laboratory Animal, National Institute of Infectious Diseases, Tokyo, Japan handlers [3]. Asymptomatic infections related to sapovirus shedding in feces have also been identified [2-4]. Sapoviruses are non-enveloped, positive-sense, single-stranded RNA viruses that encode multiple nonstructural proteins, and major and minor structural proteins [1]. The region encoding the major structural protein (VP1) is the most variable in the sapovirus genome [5] and is used for genetic typing [6]. Human sapoviruses are currently assigned to one of four genogroups (GI, GII, GIV, and GV) and are further classified into at least 18 genotypes (GI.1-7, GII.1-8, GIV.1, and GV.1-2) based on their VP1 sequences [7]. Nucleic acid detection methods, especially reverse transcription (RT)polymerase chain reaction (PCR) and real-time RT-PCR assays, are widely used for the screening of human sapoviruses [1]; however, the application of primer-independent metagenomic sequencing approaches for identification of human sapoviruses has also been reported recently [8-10]. Multiple primer sets for RT-PCR have been designed to detect human sapoviruses [1], but their reactivity with different human sapovirus genotypes has not been experimentally demonstrated except in our recent real-time RT-PCR study [11]. In the current study, we tested 11 PCR assays targeting the VP1 region of human sapoviruses, using eight published [12-15] and three newly designed/modified primer combinations and showed that only four of these PCR assays detected all 18 currently known human sapovirus genotypes (GI.1-7, GII.1-8, GIV.1, and GV.1-2). 
For the initial reactivity test, double-stranded DNA fragments (gBlocks ${ }^{\circledR}$ Gene Fragments) including the sequence targeted by the PCR primers (approximately $1700 \mathrm{bp}$ each) of the human sapovirus genotypes GI.1 (GenBank accession number X86560), GI.2 (AB614356), GI.3 (AB623037), GI.4 (AJ606693), GI.5 (DQ366345), GI.6 (AJ606694), GI.7 (AB522390), GII.1 (AJ249939), GII.2 (AY237420), GII.3 (AB630068), GII.4 (AB522397), GII.5 (LC190463), GII.6 (AY646855), GII.7 (AB630067), GII.8 (KX894315), GIV.1 (DQ058829), GV.1 (DQ366344), and GV.2 (AB775659) were synthesized (Integrated DNA Technologies, Coralville, IA), and $1.0 \times 10^{4}$ copies of these fragments were used.

To confirm the reactivity against clinical specimens, random-hexamer-primed cDNA synthesized from stool suspensions positive for sapoviruses (GI.1-7, GII.1-5 and GII.7 and GII.8, GIV.1, and GV.1-2) as well as stool suspensions positive for noroviruses (GI, GII), astrovirus types $\mathrm{I}$ and $\mathrm{IV}$, and rotavirus groups $\mathrm{A}$ and $\mathrm{C}$ and DNA purified from stool suspensions positive for adenovirus type 40/41 that had been used in a previous study and stored at $-30^{\circ} \mathrm{C}$ [11], were tested.

PCR assays were performed using $20 \mu \mathrm{L}$ of a reaction mixture containing $1 \mu \mathrm{L}$ of synthetic double-stranded DNA or $2 \mu \mathrm{L}$ of cDNA or DNA from clinical specimens, $10 \mu \mathrm{L}$ of KAPA2G Fast HotStart ReadyMix with dye (KAPA Biosystems, Wilmington, MA), and 10 pmol of each primer. PCR amplification was performed using a GeneAmp PCR System 2700 (Applied Biosystems, Foster City, CA) under the following conditions: initial denaturation at $95^{\circ} \mathrm{C}$ for 5 min, followed by 50 cycles of $95^{\circ} \mathrm{C}$ for $20 \mathrm{~s}, 48^{\circ} \mathrm{C}$ or $53^{\circ} \mathrm{C}$ for $20 \mathrm{~s}$, and $72^{\circ} \mathrm{C}$ for $5 \mathrm{~s}$, and a final extension at $72^{\circ} \mathrm{C}$ for 1 $\mathrm{min}$. The PCR reaction time was approximately $1.5 \mathrm{~h}$.

Seven $\mu \mathrm{L}$ of each PCR reaction mixture was analyzed by electrophoresis in a $2 \%$ agarose gel containing $40 \mathrm{mM}$ Tris-acetate, $1 \mathrm{mM}$ EDTA and $0.5 \mu \mathrm{g}$ of ethidium bromide per $\mathrm{mL}$, and the gel image was captured using a FAS V Gel Documentation System (Nippon Genetics, Tokyo, Japan).

Eleven PCR assays with different primer combinations were performed using a synthetic DNA panel. As shown in Fig. 1A (left panels), seven of the PCR assays, including those using the primers SV-F11 and SV-R1; SV-F21 and SV-R2; SLV5317 and SLV5749; SaV124F, SaV-1F, SaV-5F, SV-R13, and SV-R14; SaV1245Rfwd and SV-R2; SV-F13, SV-F14, SV-R13, and SV-R14; and SV-F22 and SV-R2 [12-15], either did not amplify all of the human sapovirus genotypes tested or generated PCR products with unusual sizes (i.e., GI.5 sapovirus with the SV-F11 and SV-R1 primer set) under the test conditions.

In contrast, four assays including the primer combinations i) SV-F13, SV-F14, SV-G1-R, SV-G2-R, SV-G4-R, and SV-G5-R [13], ii) M13F-SaV1245Rfwd, M13R-SV-G1-R, M13R-SV-G2-R, M13R-SV-G4-R, and M13R-SV-G5-R, iii) M13F-HuSaV-F1, M13F-HuSaV-F2, M13F-HuSaV-F3, and M13R-HuSaV-5498R, and iv) M13F-HuSaV-5159F and M13R-HuSaV-5498R amplified all the tested human sapovirus genotypes (Fig. 1A, right panels).

As shown in Fig. 1B, these four PCR assays gave similar results when cDNA prepared from sapovirus-positive clinical specimens ranging from approximately $10^{7}$ to $10^{2}$ copies/reaction (Ct value range: 18-37/reaction determined by real-time RT-PCR) representing the 17 human sapovirus genotypes (GI.1-7, GII.1-5 and GII.7 and GII.8, GIV.1, and GV.1-2) was used [11]. Samples with $\mathrm{Ct}$ values $>30$ (approximately $10^{2}$ to $10^{3}$ copies/reaction) were only slightly detectable or difficult to detect, depending on the assay used.

No cross-reactivity was observed with specimens that were positive for norovirus GI and GII, human astrovirus types I and IV, rotavirus A and C, or adenovirus type 40/41 in the four PCR assays (data not shown).

The locations of the primers used for the four PCR assays are shown in Fig. 2.

The PCR assay including six primers (SV-F13, SV-F14, SV-G1-R, SV-G2-R, SV-G4-R, and SV-G5-R) reported by Okada et al. was originally designed to generate PCR products of different lengths, depending on the genogroup: GI (500 bp), GII (430 bp), GIV (360 bp), and GV (290 bp) [13] (Fig. 1A, right panel, Fig. 1B, Fig. 2). This assay has been used in several studies to detect sapoviruses in fecal specimens [16-18]. In the current study, we confirmed that these primer combinations detected all 18 human sapovirus genotypes (GI.1-7, GII.1-8, GIV.1, and GV.1-2) using cDNA and/or synthetic DNA (Fig. 1A, right panel, and Fig. 1B).

Three other new PCR assays that amplified all 18 human sapovirus genotypes (GI.1-7, GII.1-8, GIV.1, and GV.1-2) (Fig. 1A, right panel, and Fig. 1B) included 5'-tailed primers with an 18-nt M13 sequence tag (M13F: 5'-tgtaaaacgacggccagt-3' for each forward primer and M13R: 5'-caggaaacagctatgacc- $3^{\prime}$ for each reverse primer) as a consensus anchor matrix for PCR and Sanger sequencing (Table 1).

The PCR assay including five primers (M13F-SaV1245Rfwd, M13R-SV-G1-R, M13R-SV-G2-R, M13R-SV-G4-R, and M13R-SV-G5-R) also generated PCR products of different sizes depending on the genogroup: GI (450 bp), GII (380 bp), GIV (310 bp), and GV (240 bp) (Fig. 1A, right panel, Fig. 1B, and Fig. 2).

The assay including four primers (M13F-HuSaV-F1, M13F-HuSaV-F2, M13F-HuSaV-F3, and M13R-HuSaV5498R) amplified similar-sized PCR products (approximately $460 \mathrm{bp}$ ) independently of the genogroup and genotype (Fig. 1A, right panel, and Fig. 1B). The target regions of HuSaV-F1, -F2, and -F3 recently designed as forward primers in a broadly reactive real-time PCR for human sapoviruses [11] are similar to those of SV-F13 and SV-F14 (Fig. 2), and the sapovirus-specific sequence of M13RHuSaV5498R (5'-CCCCANCCNGCVHACAT-3') (Table 1) has the same target as the SVR-DS5 (5'-CCCCACCCKGCC 
A SV-F11/SV-R1 $\left(48^{\circ} \mathrm{C}\right)$

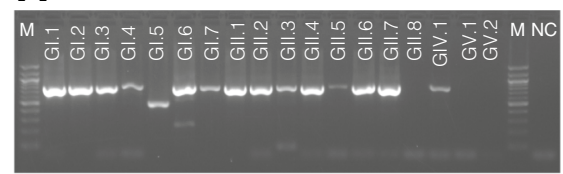

SV-F21/SV-R2 $\left(48^{\circ} \mathrm{C}\right)$

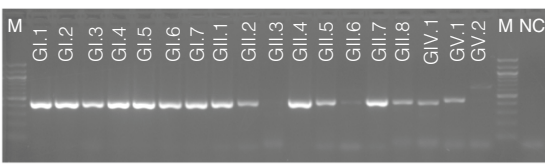

SLV5317/SLV5749 $\left(53^{\circ} \mathrm{C}\right)$

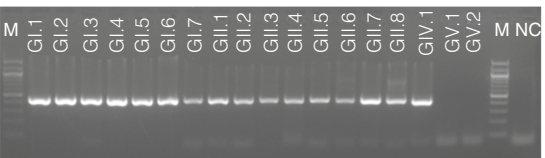

SaV124F, -1F, -5F/SV-R13,-14 $\left(48^{\circ} \mathrm{C}\right)$

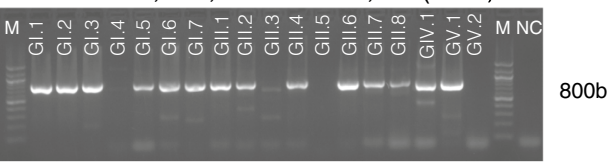

SaV1245Rfwd/SV-R2 $\left(48^{\circ} \mathrm{C}\right)$

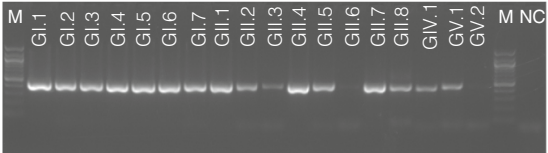

SV-F13,-F14/SV-R13,-14 $\left(48^{\circ} \mathrm{C}\right)$

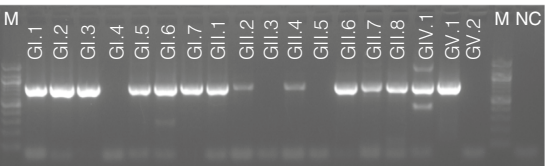

SV-F22/SV-R2 $\left(48^{\circ} \mathrm{C}\right)$

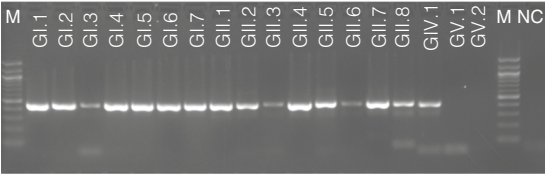

SV-F13,-F14/SV-G1, -G2, -G4,-G5R (48 $\left.{ }^{\circ} \mathrm{C}\right)$

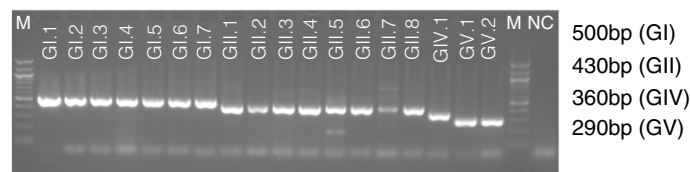

M13F-SaV1245Rfwd/M13R-SV-G1, -G2, -G4,-G5R (53 $\left.{ }^{\circ} \mathrm{C}\right)$

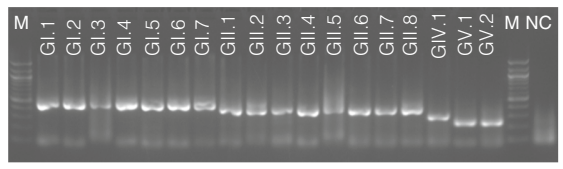

$450 \mathrm{bp}$ (Gl) $380 \mathrm{bp}$ (GII) $310 \mathrm{bp}$ (GIV) 240bp (GV)

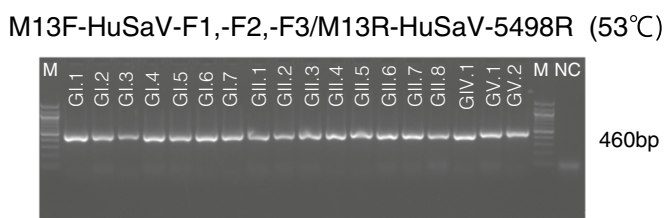

M13F-HuSaV-5159F/M13R-HuSaV-5498R $\left(53^{\circ} \mathrm{C}\right)$

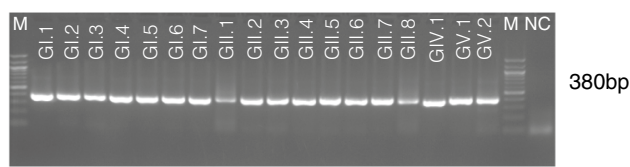

B SV-F13,-F14/SV-G1, -G2, -G4,-G5R $\left(48^{\circ} \mathrm{C}\right)$

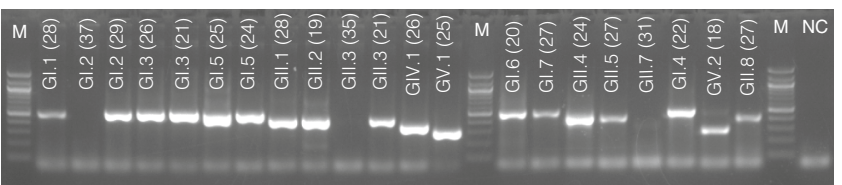

M13F-HuSaV-F1,-F2,-F3/M13R-HuSaV-5498R (53ㄷ)
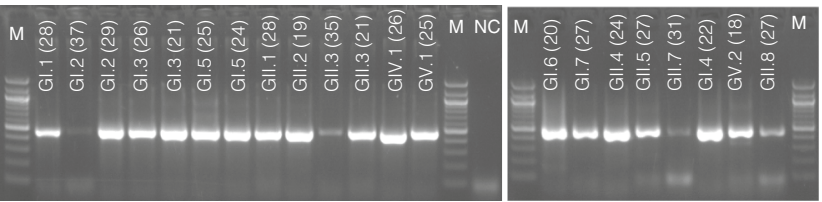

Fig. 1 Reactivity of PCR assays with different human sapovirus genotypes. Agarose gel electrophoresis images of the PCR products amplified from (A) synthetic double-stranded DNA from GI.17, GII.1-8, GIV.1, and GV.1-2 sapoviruses; (B) cDNA from GI.1-7, GII.1-5 and GII.7-8, GIV.1, and GV.1-2 sapoviruses. Ct values of each sample measured by real-time RT-PCR in a previous study [11]
M13F-SaV1245Rfwd/M13R-SV-G1, -G2, -G4,-G5R (53ํ)

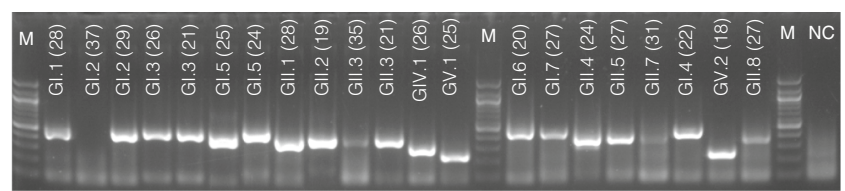

M13F-HuSaV-5159F/M13R-HuSaV-5498R $\left(53^{\circ} \mathrm{C}\right)$

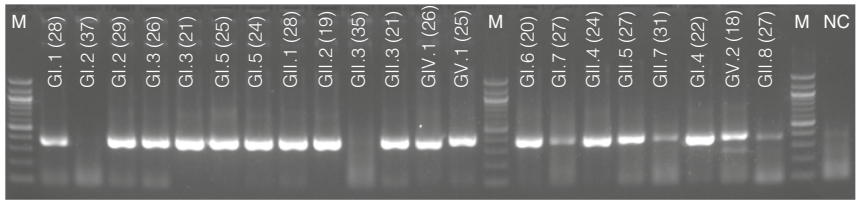

are shown in parentheses. The primer combinations and PCR product size(s) are indicated at the top and right side, respectively, of each gel image. The annealing temperature is indicated in parentheses after the primer names. M, DNA size marker 100 bp DNA ladder (New England Biolabs); NC, negative control without template 


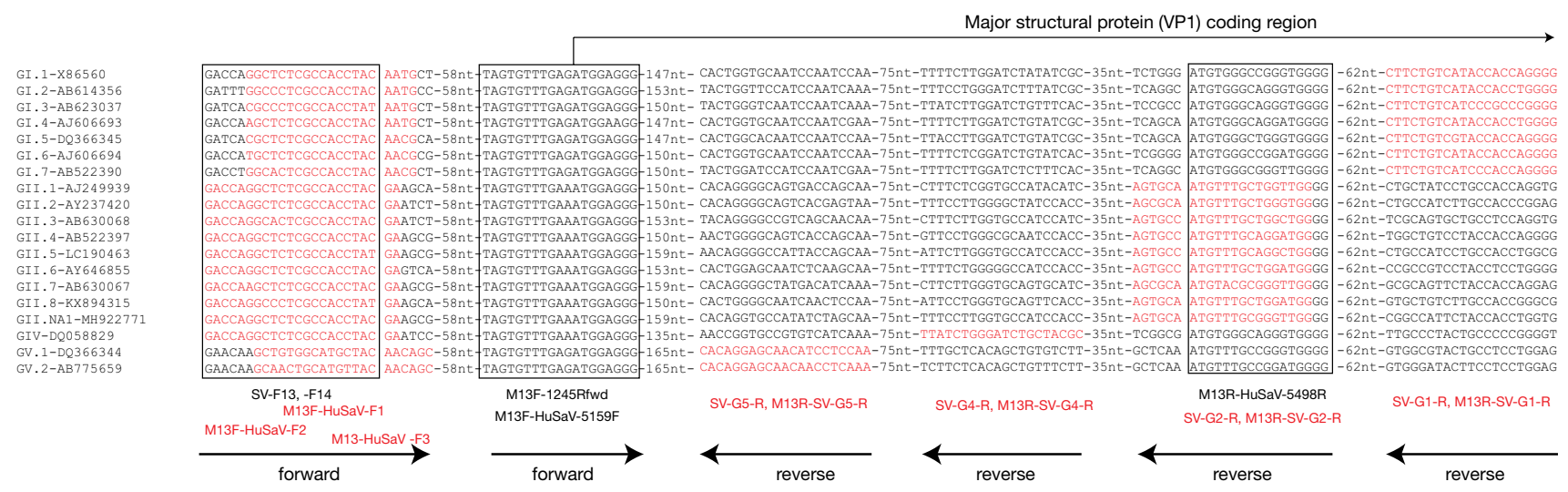

Fig. 2 Regions targeted by the primers of the four broadly reactive PCR assays for human sapoviruses. A nucleotide sequence alignment of representative human sapovirus strains corresponding to the 18 genotypes (GI.1-7, GII.1-8, GIV.1, and GV.1-2) used to synthesize DNA in this study and the newly proposed genotype GII.NA1, the locations of the primers and their targeted sequences used for the four broadly reactive PCR assays, and the GenBank accession numbers of the representative strains of each genotype are shown. The target region sequences of SV-F13, -F14 and M13F-1245Rfwd, and M13-HuSaV-5159F and M13-HuSaV-5498R are boxed, and those of M13F-HuSaV-F1, F2, -F3, and SV-G1-R, -G2-R, -G4-R, -G5R with or without an M13 sequence tag are labeled in red

Table 1 Primer combinations for amplification of the 18 human sapovirus genotypes

\begin{tabular}{|c|c|c|c|c|c|}
\hline Primer name & Sequence ( $5^{\prime}$ to $\left.3^{\prime}\right)$ & Di Direction & $\begin{array}{l}\text { Location in } \\
\text { genome with } \\
\text { accession nu }\end{array}$ & $\begin{array}{l}\text { he sapovirus } \\
\text { GenBank } \\
\text { mber }\end{array}$ & Reference \\
\hline SV-F13 & GAY YWG GCY CTC GCY ACC TAC & Forward & $5074-5094$ & X86560 & 13 \\
\hline SV-F14 & GAA CAA GCT GTG GCA TGC TAC & Forward & $5074-5094$ & X86560 & \\
\hline SV-G1-R & CCC BGG TGG KAY GAC AGA AG & Reverse & $5561-5580$ & X86560 & \\
\hline SV-G2-R & CCA NCC AGC AAA CAT NGC RCT & Reverse & $5483-5503$ & AY237420 & \\
\hline SV-G4-R & GCG TAG CAG ATC CCA GAT AA & Reverse & $5413-5432$ & DQ058829 & \\
\hline SV-G5-R & TTG GAG GWT GTT GCT CCT GTG & Reverse & $5384-5404$ & AY646856 & \\
\hline M13F-SaV 1245Rfwd & tgtaaaacgacggccagt TAG TGT TTG ARA TGG AGG G & Forward & $5159-5177$ & X86560 & This study \\
\hline M13R-SV-G1-R & caggaaacagctatgacc CCC BGG TGG KAY GAC AGA AG & Reverse & $5561-5580$ & X86560 & \\
\hline M13R-SV-G2-R & caggaaacagctatgacc CCA NCC AGC AAA CAT NGC RCT & Reverse & $5483-5503$ & AY237420 & \\
\hline M13R-SV-G4-R & caggaaacagctatgacc GCG TAG CAG ATC CCA GAT AA & Reverse & $5413-5432$ & DQ058829 & \\
\hline M13R-SV-G5-R & caggaaacagctatgacc TTG GAG GWT GTT GCT CCT GTG & Reverse & $5384-5404$ & AY646856 & \\
\hline M13F-HuSaV-F1 & tgtaaaacgacggccagt GGC HCT YGC CAC CTA YAA YG & Forward & $5079-5098$ & X86560 & This study \\
\hline M13F-HuSaV-F2 & tgtaaaacgacggecagt GAC CAR GCH CTC GCY ACC TAY GA & Forward & $5078-5100$ & AJ249939 & \\
\hline M13F-HuSaV-F3 & tgtaaaacgacggccagt GCW RYK GCW TGY TAY AAC AGC & Forward & $5121-5141$ & DQ366344 & \\
\hline M13R-HuSaV-5498R & caggaaacagctatgacc CCC CAN CCN GCV HAC AT & Reverse & $5482-5498$ & X86560 & \\
\hline M13F-HuSaV-5159F & tgtaaaacgacggccagt TAG TGT TTG ARA TGG ARG G & Forward & $5159-5177$ & X86560 & This study \\
\hline M13R-HuSaV-5498R & caggaaacagctatgacc CCC CAN CCN GCV HAC AT & Reverse & $5482-5498$ & X86560 & \\
\hline
\end{tabular}

CACAT-3') and SVR-DS6 (5'-CCCCAMCCMGCMMACAT-3') primers reported by Sano et al. [19].

The assay including two primers (M13F-HuSaV-5159F and M13R-HuSaV-5498R) generated similar-sized PCR products (approximately $380 \mathrm{bp}$ ) for all of the sapovirus genotypes tested (Fig. 1A, right panel, and Fig. 1B). The sapovirus-specific sequence of M13F-HuSaV-5159F was identical to that of 1245Rfwd [15] except for $1 \mathrm{nt}$ (Fig. 2 and Table 1), and they shared the same complementary sequence in the reverse primers (HuSaV-R, and SaV1245R, respectively) used in real-time PCR assays for detection of human sapoviruses $[5,11]$.

In primer-dependent PCR assays, the selection of broadly reactive primer sets is important for detecting genetically diverse human sapoviruses. In this study, seven out of 11 primer combinations, including widely used primer sets, did not detect all of the sapovirus genotypes tested (Fig. 1A and B). This would cause a bias towards the detected strains or 
cause several genotypes to be missed when used for surveillance. We recently reported an improved broad-range real-time RT-PCR assay that detected all 18 of the currently identified human sapovirus genotypes [11]. This assay can be used as a screening tool, but the amplicon size (approximately $100 \mathrm{bp}$ ) is too short for further genetic characterization by sequence analysis. Selection and combination of the human-sapovirus-targeting, broad-range RT-PCR assays described in this report can be used to confirm real-time RT-PCR results and an alternative screening tool to identify genetically diverse human sapoviruses in samples. An assay that distinguishes genogroups based on PCR product size will be useful in cases in which a sample includes sapoviruses of different genogroups. New sapovirus strains assigned as GII.NA1 were reported recently [20]. Based on their nucleotide sequences, the primers used in the four broadly reactive PCR assays would probably also recognize this new candidate genotype (Fig. 2 and Table 1), although experimental confirmation is required.

Funding This work was supported by a grant from the Research Program on Emerging and Re-emerging Infectious Diseases from the Japan Agency for Medical Research and Development (AMED) (JP19fk0108049 and JP20fk0108139). The funding body had no role in study design, analysis and interpretation of data, or the decision to submit the article for publication.

\section{Compliance with ethical standards}

Conflict of interest There are no conflicts of interest among the authors.

Ethics approval The ethics committee of the National Institute of Infectious Diseases deemed that human subject regulations did not apply to this study because it only used extracted and stored virus nucleic acids (Receipt number 1113).

\section{References}

1. Oka T, Wang Q, Katayama K, Saif LJ (2015) Comprehensive review of human sapoviruses. Clin Microbiol Rev 28(1):32-53. https://doi.org/10.1128/CMR.00011-14

2. Hebbelstrup Jensen B, Jokelainen P, Nielsen ACY, Franck KT, Rejkjaer Holm D, Schonning K, Petersen AM, Krogfelt KA (2019) Children attending day care centers are a year-round reservoir of gastrointestinal viruses. Sci Rep 9(1):3286. https://doi. org/10.1038/s41598-019-40077-9

3. Kobayashi S, Fujiwara N, Yasui Y, Yamashita T, Hiramatsu R, Minagawa H (2012) A foodborne outbreak of sapovirus linked to catered box lunches in Japan. Arch Virol 157(10):1995-1997. https://doi.org/10.1007/s00705-012-1394-8

4. Yoshida T, Kasuo S, Azegami Y, Uchiyama Y, Satsumabayashi K, Shiraishi T, Katayama K, Wakita T, Takeda N, Oka T (2009) Characterization of sapoviruses detected in gastroenteritis outbreaks and identification of asymptomatic adults with high viral load. J Clin Virol 45(1):67-71. https://doi.org/10.1016/j. jcv.2009.03.003

5. Oka T, Katayama K, Hansman GS, Kageyama T, Ogawa S, Wu FT, White PA, Takeda N (2006) Detection of human sapovirus by real-time reverse transcription-polymerase chain reaction. J Med Virol 78(10):1347-1353. https://doi.org/10.1002/ jmv.20699

6. Oka T, Mori K, Iritani N, Harada S, Ueki Y, Iizuka S, Mise K, Murakami K, Wakita T, Katayama K (2012) Human sapovirus classification based on complete capsid nucleotide sequences. Arch Virol 157(2):349-352. https://doi.org/10.1007/s0070 5-011-1161-2

7. Diez-Valcarce M, Castro CJ, Marine RL, Halasa N, Mayta H, Saito M, Tsaknaridis L, Pan CY, Bucardo F, Becker-Dreps S, Lopez MR, Magana LC, Ng TFF, Vinje J (2018) Genetic diversity of human sapovirus across the Americas. J Clin Virol 104:65-72. https://doi.org/10.1016/j.jcv.2018.05.003

8. Li W, Dong S, Xu J, Zhou X, Han J, Xie Z, Gong Q, Peng H, Zhou C, Lin M (2020) Viral metagenomics reveals sapoviruses of different genogroups in stool samples from children with acute gastroenteritis in Jiangsu, China. Arch Virol 165(4):955-958. https://doi.org/10.1007/s00705-020-04549-y

9. Hergens MP, Nederby Ohd J, Alm E, Askling HH, Helgesson S, Insulander M, Lagerqvist N, Svenungsson B, Tihane M, Tolfvenstam T, Follin P (2017) Investigation of a food-borne outbreak of gastroenteritis in a school canteen revealed a variant of sapovirus genogroup V not detected by standard PCR, Sollentuna, Sweden, 2016. Euro Surveill. https://doi.org/10.2807/1560-7917. es.2017.22.22.30543

10. Shibata S, Sekizuka T, Kodaira A, Kuroda M, Haga K, Doan YH, Takai-Todaka R, Katayama K, Wakita T, Oka T, Hirata H (2015) Complete genome sequence of a novel GV.2 sapovirus strain, NGY-1, detected from a suspected foodborne gastroenteritis outbreak. Genome Announc. https://doi.org/10.1128/genomea.01553 $-14$

11. Oka T, Iritani N, Yamamoto SP, Mori K, Ogawa T, Tatsumi C, Shibata S, Harada S, Wu FT (2019) Broadly reactive real-time reverse transcription-polymerase chain reaction assay for the detection of human sapovirus genotypes. J Med Virol 91(3):370 377. https://doi.org/10.1002/jmv.25334

12. Okada M, Shinozaki K, Ogawa T, Kaiho I (2002) Molecular epidemiology and phylogenetic analysis of Sapporo-like viruses. Arch Virol 147(7):1445-1451. https://doi.org/10.1007/s0070 5-002-0821-7

13. Okada M, Yamashita Y, Oseto M, Shinozaki K (2006) The detection of human sapoviruses with universal and genogroup-specific primers. Arch Virol 151(12):2503-2509. https://doi.org/10.1007/ s00705-006-0820-1

14. Yan H, Yagyu F, Okitsu S, Nishio O, Ushijima H (2003) Detection of norovirus (GI, GII), Sapovirus and astrovirus in fecal samples using reverse transcription single-round multiplex PCR. J Virol Methods 114(1):37-44. https://doi.org/10.1016/j.jviro met.2003.08.009

15. Kitajima M, Oka T, Haramoto E, Katayama H, Takeda N, Katayama K, Ohgaki S (2010) Detection and genetic analysis of human sapoviruses in river water in Japan. Appl Environ Microbiol 76(8):2461-2467. https://doi.org/10.1128/AEM.02739-09

16. Harada S, Okada M, Yahiro S, Nishimura K, Matsuo S, Miyasaka J, Nakashima R, Shimada Y, Ueno T, Ikezawa S, Shinozaki K, Katayama K, Wakita T, Takeda N, Oka T (2009) Surveillance of pathogens in outpatients with gastroenteritis and characterization of sapovirus strains between 2002 and 2007 in Kumamoto Prefecture, Japan. J Med Virol 81(6):1117-1127. https://doi. org/10.1002/jmv.21454

17. Pang XL, Lee BE, Tyrrell GJ, Preiksaitis JK (2009) Epidemiology and genotype analysis of sapovirus associated with 
gastroenteritis outbreaks in Alberta, Canada: 2004-2007. J Infect Dis 199(4):547-551. https://doi.org/10.1086/596210

18. Harada S, Oka T, Tokuoka E, Kiyota N, Nishimura K, Shimada Y, Ueno T, Ikezawa S, Wakita T, Wang Q, Saif LJ, Katayama K (2012) A confirmation of sapovirus re-infection gastroenteritis cases with different genogroups and genetic shifts in the evolving sapovirus genotypes, 2002-2011. Arch Virol 157(10):1999-2003. https://doi.org/10.1007/s00705-012-1387-7

19. Sano D, Perez-Sautu U, Guix S, Pinto RM, Miura T, Okabe S, Bosch A (2011) Quantification and genotyping of human sapoviruses in the Llobregat river catchment, Spain. Appl Environ Microbiol 77(3):1111-1114. https://doi.org/10.1128/AEM.01721 $-10$
20. Diez-Valcarce M, Montmayeur A, Tatusov R, Vinje J (2019) NearComplete Human Sapovirus Genome Sequences from Kenya. Microbiol Resour Announc. https://doi.org/10.1128/mra.01602 $-18$

Publisher's Note Springer Nature remains neutral with regard to jurisdictional claims in published maps and institutional affiliations. 